\title{
Sebusuppressive efficacy of the antioxidant bis-ethylhexyl hydroxydimethoxy benzylmalonate in the treatment of oily and blemished skin
}

This article was published in the following Dove Press journal:

Clinical, Cosmetic and Investigational Dermatology

17 August 2012

Number of times this article has been viewed

\section{Nicole Gerlach' \\ Ruediger Graf ${ }^{2}$ \\ Gabriele Witte ${ }^{2}$ \\ Marina Lefort ${ }^{2}$ \\ Frank Pfluecker ${ }^{2}$ \\ Ulrike Heinrich' \\ Hagen Tronnier'}

'DermaTronnier, University of Witten-Herdecke, Witten, Germany;

${ }^{2}$ Merck KGaA, Darmstadt, Germany
Correspondence: Nicole Gerlach

DermaTronnier, Alfred Herrhausen-Str 44, 58455, Witten, Germany

Email nicole.gerlach@uni-wh.de

\begin{abstract}
The aim of this study was to assess the effect of 2\% bis-ethylhexyl hydroxydimethoxy benzylmalonate (HDBM; RonaCare ${ }^{\circledR}$ AP) as an active ingredient in the treatment of oily and blemished skin. This study was carried out as a randomized, placebo-controlled, single-blind study on 44 test subjects with blemished skin over a period of 8 weeks. Sebum measurement, skin inflammation/redness scoring by an expert, photographic documentation and a self-assessment with regard to skin improvement, and tests of skin compatibility and galenic characteristics were performed. Treatment with $2 \%$ HDBM resulted in a significant reduction of sebum excretion and showed efficacy against inflamed/red lesions also shown by photographic documentation. Efficacy and galenic performance of $2 \%$ HDBM were judged to be superior to the placebo emulsion. Additionally, 2\% HDBM was well tolerated; approximately $80 \%$ of the test subjects rated the compatibility as good to very good.
\end{abstract}

Keywords: oily and blemished skin, sebum excretion, antiseborrheic, anti-inflammatory, bis-ethylhexyl hydroxydimethoxy benzylmalonate (HDBM)

\section{Introduction}

Blemished skin is the most common skin condition worldwide and commonly affects the face (forehead, cheek, and chin), chest, and back, where sebaceous gland hair follicles are mostly found. Due to hormonal changes approximately $90 \%$ of teenagers and young adults experience some form of blemished skin, with more boys than girls being affected. ${ }^{1}$ Peak incidence is between 14-17 years of age for females and between 16-19 years of age for males. ${ }^{2}$ However, after puberty blemished skin gradually declines with age. The blemished skin or pre-acne is primarily characterized by open (blackhead) or closed (whitehead) comedones as well as shiny or irritated skin areas, whereas acne shows comedones, pimples, papules, and pustules. The sine qua non of providing a suitable treatment for blemish-prone skin is the knowledge of its pathogenesis. Skin tending to blemishes is understood as a complex condition in which the skin is subject to multiple pathogenic alterations. The four key pathogenic factors leading to moist, oily skin, to pre-acne, and potentially to acne are follicular epithelial hyperproliferation and hyperkeratinization of the follicular duct, excess sebum, ${ }^{3-6}$ and release of inflammatory mediators and bacterial colonization of the hair follicle by Propionibacterium acnes. Furthermore, genetics ${ }^{7}$ and extrinsic factors like medications, stress, ${ }^{8}$ and products used on the skin ${ }^{9}$ are also responsible for blemished skin.

Sebum, consisting of triglyceride, fatty acids, wax esters, squalene, and cholesterol, is thought to play the major role in blemished skin. In particular, the peroxidation of squalene has been shown to be involved in the initiation and development of acne lesions. ${ }^{10}$ 
Squalene, one of the major sebum lipids of human skin surface is prone to oxidative attack by pollutants and sun exposure. The peroxidation process resulting in the formation of squalene monohydroperoxide could be mainly attributed to aggression by singlet oxygen. ${ }^{11}$ Thus, the protection of the sebum components from oxidizing appears to be a possible strategy to inhibit one of the sources of acne emergence. More specifically, the use of effective singlet oxygen quenchers could be particularly relevant to support this purpose.

Based on these facts, bis-ethylhexyl hydroxydimethoxy benzylmalonate (HDBM) with its powerful antioxidant activity as demonstrated in several antioxidant tests, ${ }^{12}$ might be a promising candidate in the treatment of blemished skin. With four hydrogen equivalents per molecule, it displays a great reducing power, being able to scavenge free radicals as well as other reactive species generated by UV irradiation. ${ }^{13}$ Its oxidized counterpart, HDBMox, also works as a singlet oxygen quencher ${ }^{14}$ so that with the tandem of HDBM-HDBMox, a dynamic and long-lasting antioxidative protection is built up. The outstanding broadband antioxidative properties of HDBM suggest that HDBM may exhibit a protecting effect on sebum squalene. As a result, the pro-inflammatory activity of squalene oxidation products would be inhibited. This, in turn, would improve the overall appearance of skin tending to acne.

Thus, the objective of the present study is to evaluate the topical impact of HDBM on conditions connected to blemished skin, especially its ability to reduce skin oiliness and skin inflammation/redness.

\section{Materials and methods Test products}

The test products involve a placebo emulsion and a verum emulsion with $2 \%$ HDBM. The oil in water emulsions were produced according to the following procedure with the ingredients listed in Table 1.

The first step of this cold processing is the preparation of phase A. Phase B was added to phase A and the batch was stirred until homogeneous. Phase $\mathrm{C}$ was combined and introduced to A/B while stirring. The emulsion was homogenized. Finally, the perfuming oil was added while stirring.

\section{Single controlled patch test to determine dermal toxicity}

The tested substances (placebo and verum emulsion) were applied to the back of 50 adult test subjects. The test group was composed of one third of test subjects with sensitive skin, one third of atopic test subjects and one third of test subjects with normal skin. The quantity of the applied test substance was approximately $2 \mathrm{mg}$, placed on an $8 \mathrm{~mm}$ diameter test patch (Curatest ${ }^{\circledR}$, Lohmann and Rauscher, Neuwied, Rhineland-Palatinate, Germany). After 48 hours, the test patch was removed and the effect on the skin was assessed after approximately 15-20 minutes. Further readings of possible skin reactions were taken 72 hours and 96 hours after the test patch application. The visual assessment was based on the following evaluation score: $0=$ no reaction, $\pm=$ doubtful erythema, $+=$ clearly visible erythema, $++=$ strong erythema and/or papules, $+++=$ strong erythema plus dense papules and/ or vesicles, $++++=$ strong erythema plus bulla or necrosis.

\section{Study design}

The study was carried out as a randomized, placebo controlled, single-blind study on 44 test subjects of both sexes, 15-28 years of age (Table 2). The subjects were selected according to the inclusion criteria, which were oily or combined type of skin with a sebum level of $75-100 \mu \mathrm{g} / \mathrm{cm}^{2}$ and a skin inflammation/redness score of at least 2 or with

Table I O/W test emulsions placebo vs verum

\begin{tabular}{llll}
\hline Phase & Ingredients (INCI) & Placebo & Verum \\
\cline { 2 - 3 } & & {$[\%]$} & {$[\%]$} \\
\hline A & Bis-ethylhexyl hydroxydimethoxy benzylmalonate & 2.00 \\
& Cyclopentasiloxane, & 0.00 & \\
& cyclohexasiloxane & 5.00 & \\
& Diisopropyl adipate & 7.00 & 7.00 \\
& Paraffinum liquidum (mineral oil) & 2.00 & 2.00 \\
B & Phenoxyethanol, ethylhexyl glycerin & 1.00 & 1.00 \\
C & Polysorbate 20, polyacrylate I3, polyisobutene & 1.50 & 1.50 \\
& Aqua (water) & 80.35 & 78.35 \\
& Disodium edta & 0.05 & 0.05 \\
D & Glycerin & 3.00 & 3.00 \\
& Parfume & 0.10 & 0.10 \\
\hline
\end{tabular}

Abbreviation: O/W, oil in water. 
Table 2 Sex, age, and skin type distribution of test subjects

\begin{tabular}{llll}
\hline & Total & Male & Female \\
\hline Number & 44 & 15 & 29 \\
Average age & 19.9 & 19.5 & 20.3 \\
Age limit & $15-28$ & $15-27$ & $15-28$ \\
$\begin{array}{l}\text { Skin type } \\
\quad \text { Oily }\end{array}$ & 13 & 3 & \\
$\quad$ Combination & 21 & 12 & 10 \\
Thereof & & & 19 \\
$\quad$ Sensitive & 6 & 1 & \\
$\quad$ Atopic & 4 & 2 & 5 \\
Both & 5 & 2 & 2 \\
\hline
\end{tabular}

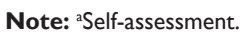

sebum levels over $100 \mu \mathrm{g} / \mathrm{cm}^{2}$ on the forehead and a skin inflammation/redness score of at least one. Exclusion criteria were medications which affect the skin response such as antibiotics and anti-inflammatory medications and the regular use of sun beds. The subjects read the information sheets and signed the informed consent prior to study participation and promised not to use any other acne treatment products and to maintain their skin cleansing habits. The study design for non-invasive surveys was approved by the Ethics Committee of the University of Witten-Herdecke, Germany.

The test subjects applied the emulsion twice a day - in the morning and evening. The duration of the study was 8 weeks. The effectiveness of 2\% HDBM was evaluated at baseline, after 4 weeks as a midpoint evaluation, and after 8 weeks of use by the investigator and by the test subjects themselves through a self-evaluation.

\section{Application and compatibility test}

For the assessment of the test product, the test subjects evaluated the galenic cosmetic acceptance, skin compatibility, and efficacy. All parameters were rated on a 6-point scale, where 1 denotes the highest grade and 6 the lowest. Finally, a mean score value of all parameters was calculated to state the overall cosmetic appearance. Clinical side effects like redness, swelling, desquamation, and pimples, and sensory effects like burning, tense feeling, and itching were recorded by the test subjects. The intensity of the side effects was rated on a scale from one to five: $0=$ no reaction, $1=$ very slight reaction, $2=$ slight reaction, $3=$ moderate reaction, $4=$ intense reaction, $5=$ very intense reaction. In addition, the duration of side effects was recorded.

\section{Efficacy tests}

The sebum level on the forehead was measured using Sebumeter $^{\circledR}$ SM815 (Courage and Khazaka, Electronic GmbH, Cologne, NRW, Germany). This method is based on greasespot photometry. Measured values are given in $\mu \mathrm{g} / \mathrm{cm}^{2}$.
All subjects were required to complete a 30 minute acclimation period $\left(22^{\circ} \mathrm{C}, 40 \%\right.$ relative humidity). The values recorded were means of three measurements.

To determine the skin inflammation/redness a subjective visual evaluation of each test subject was done by an expert according to the following scale: $0=$ no irritation, $1=$ slight irritation, 2 = moderate irritation, 3 = intense irritation.

To objectively measure the responsiveness to the treatment of oily and blemished skin photographic documentation was used. Color photographs of the front, left-side, and right-side of the face were taken using the Fotofinder mediscope (Fotofinder Systems GmbH, Bad Birnbach, Bavaria, Germany). The Fotofinder mediscope allows the taking of consistent photographs for a before and after comparison. DatInf $^{\circledR}$ UVScan software (DatInf ${ }^{\circledR} \mathrm{GmbH}$, Tübingen, Bavaria, Germany) was used for the visualization of skin blemishes and skin irritations.

\section{Statistical analysis}

For all parameters pre-post differences were calculated and analyzed. Within the two groups (verum - placebo) each time point was compared to week 0 using the Wilcoxon signedrank test. The pre-post difference of the two groups was compared using the Wilcoxon signed-rank test. A $P$-value of $P<0.05$ was considered as statistically significant.

\section{Results}

\section{Patch test}

None of the 50 test subjects showed a positive or doubtful reaction to the placebo or verum emulsion. In addition, no pre-existing sensitizations were observed.

\section{Skin compatibility}

No serious adverse events (AEs) were reported and none of the participating test subjects prematurely terminated the study because of adverse events. In the placebo group a total of 17 AEs were recorded by nine test subjects (40.9\%). The AEs were burning, redness, itching, tense feeling of the skin, burning around the eyes, and in one case burning of eyes as well as watery eyes. Five of the participating test subjects experienced mostly continuous AEs of very mild to mild severity, whereas three test subjects complained about moderate AEs. A total of eleven AEs were recorded by five test subjects $(22.73 \%)$ in the verum group. The AEs were burning, redness, and tense feeling of the skin, as well as burning and tense feeling around the eyes. AEs were mostly continuous and of mild severity. In the self-assessment the skin compatibility was rated as very good to good in $77 \%$ of the verum group and in $50 \%$ of the placebo group (Figure 1). 


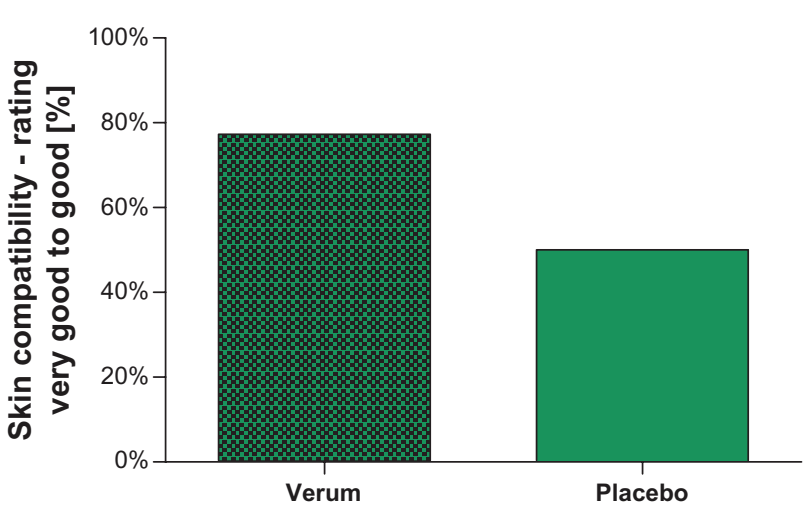

Figure I Subjects' self-assessment of skin compatibility after 8 weeks of treatment with $2 \%$ HDBM emulsion or placebo.

Notes: Shown is percentage of test subjects who rated the skin compatibility of the product or placebo as very good to good.

\section{Efficacy on sebum reduction}

Increased sebum excretion has been well-known to play an important role in the development of acne. Thus, sebum level was determined before, during, and after a topical treatment with $2 \%$ HDBM or placebo. The baseline sebum level on the forehead ranged between $75-259 \mu \mathrm{g} / \mathrm{cm}^{2}$ (mean value $161.77 \mu \mathrm{g} / \mathrm{cm}^{2}$ ) in the placebo group and between $76-258 \mu \mathrm{g} / \mathrm{cm}^{2}$ (mean value $169.88 \mu \mathrm{g} / \mathrm{cm}^{2}$ ) in the verum group, indicating a normal to oily forehead skin of all test subjects. The mean sebum level after 4 weeks of treatment with $2 \%$ HDBM declined significantly by $18.63 \%$ to $138.32 \mu \mathrm{g} / \mathrm{cm}^{2}$. Following 8 weeks of treatment, the sebum level was even more reduced by a total of $19.91 \%$ to $136.14 \mu \mathrm{g} / \mathrm{cm}^{2}$. These decreases in sebum level were statistically significant with $P$-values $<0.01$. In the placebo group the sebum level decreased slightly by $5.0 \%$ after 4 weeks and by $3.4 \%$ after 8 weeks, but was not statistically significant (Figure 2). Boxplots were used to give an overall picture of the test subjects' pre-post sebum level difference distribution. As can be seen from Figure 3, there was a negative shift in the sebum level difference after 4 weeks (median $=-33$ ) as well as 8 weeks (median $=-32$ ) in the verum group. After 8 weeks, one test subject even showed a decrease of $-160 \mu \mathrm{g} / \mathrm{cm}^{2}$ (lower whisker) and only a few test subjects showed an increase in sebum level after 4 and 8 weeks with maximum values of 81 and 72 respectively (upper whisker). In the placebo group only a slight negative shift (sebum reduction) could be observed after 4 weeks (median $=-6$ ). Decrease and increase of sebum level were normally distributed with maximum and minimum values of -99 and +72 after 4 weeks and -82 and +55 after 8 weeks. The pre-post-difference comparison of the verum versus placebo group showed no difference in sebum level, neither at week 4 nor at week 8

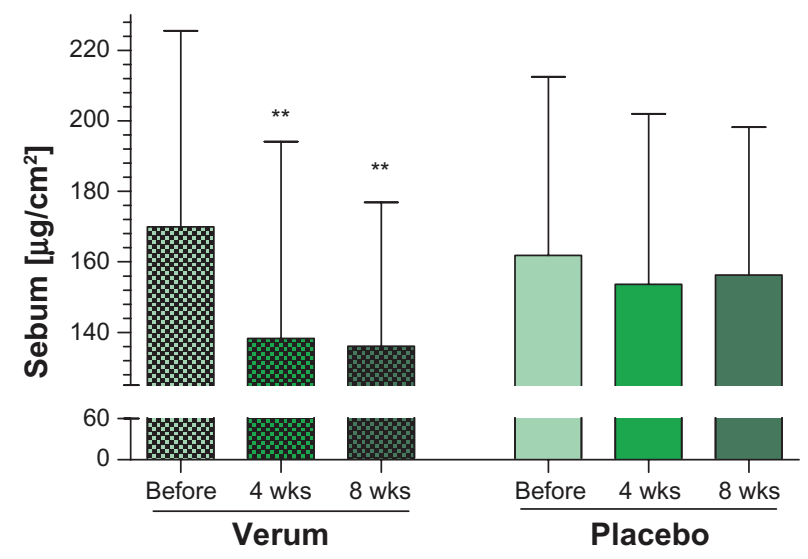

Figure 2 Sebum measurements (in $\mu \mathrm{g} / \mathrm{cm}^{2}$ ) before, after 4 weeks and 8 weeks of treatment with verum or placebo.

Notes: Statistical differences in the sebum reduction between baseline and after 4 weeks and 8 weeks of treatment are indicated $(* * P<0.01$, Wilcoxon signed-rank test). The bars indicate means \pm SD for 22 test subjects.

(Figure 3). Nevertheless, after treatment with 2\% HDBM the sebum level was significantly reduced in the verum group and thus it helps to reduce sebum excretion.

\section{Efficacy on inflammation/redness reduction}

In addition to excess sebum excretion, inflammation plays another pathogenetic role in acne. For this reason, improvements were assessed by comparing the inflammation and redness of the skin to observations made during the baseline visit by an expert.

The mean baseline inflammation/redness score was 1.95 in the verum group. After 4 weeks of treatment with $2 \%$ HDBM the inflammation/redness was improved by $9.2 \%$ to a score of 1.77. Following 8 weeks of treatment, inflammation/redness



Figure 3 Boxplot graphic illustrating the difference (pre-post) of sebum level after 4 weeks and 8 weeks of treatment compared to baseline.

Note: Median, upper and lower quartile, whiskers and suspected outliers $(\bullet)$ are illustrated. 
was further improved by a total of $25.64 \%$ to a score of 1.45 . The decrease in inflammation/redness after 8 weeks was statistically significant with a $P$-value $<0.05$. In the placebo group the mean baseline inflammation/redness score was 1.55. No statistically significant changes in inflammation/ redness were observed after 4 or 8 weeks (Figure 4). The score difference after 4 weeks was almost identical in the verum and placebo group with a median of 0 and a distribution that was negatively skewed. However, the score difference after 8 weeks showed a clear negative shift in the verum group $($ median $=-0.5)$ compared to the placebo group $($ median $=0)$. The parameter inflammation/redness showed a statistically significant pre-post-difference $(P=0.0382)$ between the groups in favor of the verum group (Figure 5). The reduction of inflammation and redness of lesions can be seen clearly in the photos of Figure 6. Thus, HDBM helped to significantly reduce inflammation/redness.

\section{Evaluation of efficacy by test subjects}

The effectiveness of the $2 \%$ HDBM treatment for blemished skin was also confirmed by a self-assessment of the test subjects. At the end point, $60 \%$ of the test subjects of the verum group agreed that their skin texture improved (mean score $=2.45$ ) and skin oiliness was reduced (mean score $=2.41$ ), $50 \%$ agreed their pimples were reduced (mean score $=2.59), 45 \%$ agreed that it prevented the formation of skin impurities and pimples (mean score $=2.72$ ), $41 \%$ noted a reduction of their blackheads (mean score $=2.76$ ), and $32 \%$ noted a tightening of their pores (mean score $=2.81$ ). Thus, the overall efficacy of the skin blemishes treatment

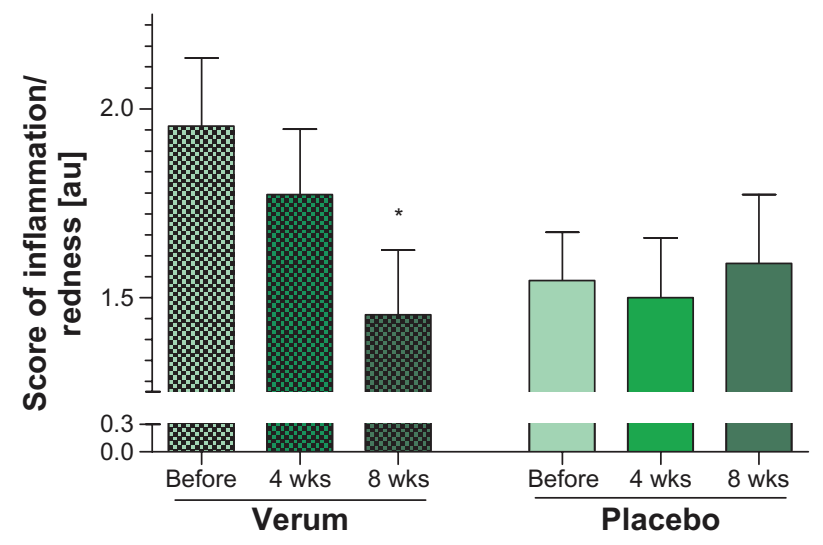

Figure 4 Inflammation and redness score before, after 4 weeks and 8 weeks of treatment with verum or placebo.

Notes: Inflammation/redness was determined according to the following scale: $0=$ no irritation, $\mathrm{I}=$ slight irritation, 2 = moderate irritation, 3 = intense irritation Statistical differences in the inflammation/redness score between baseline and after 4 weeks and 8 weeks of treatment are indicated $(* P<0.05$, Wilcoxon signed-rank test). The bars indicate means \pm SD for 22 test subjects.

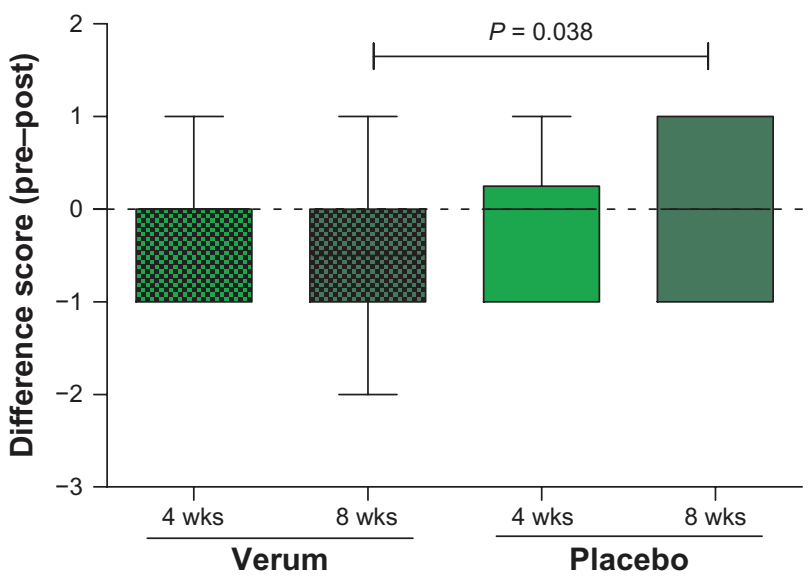

Figure 5 Boxplot graphic illustrating the difference (pre-post) of inflammation/ redness score after 4 weeks and 8 weeks of treatment compared to baseline.

Notes: Median, upper and lower quartile and whiskers are illustrated. Statistical significance in the pre-post difference of the two groups is indicated.

was rated as very good or good by $45 \%$ (mean score $=2.55)$ of the test subjects. However, compared to the verum group, $30 \%-55 \%$ fewer test subjects of the placebo group reported an improvement of their skin texture, a prevention of the formation of skin impurities and pimples, a reduction of blackheads, and a tightening of pores. Thus, the overall efficacy was rated as very good or good by only $31 \%$ (mean score $=2.95$ ) of the test subjects (Figure 7). Thus, our findings from the $2 \%$ HDBM treatment indicate an important role in treatment of blemish-prone skin.

\section{Test subjects' view of cosmetic properties}

In addition, the test subjects evaluated the cosmetic acceptance and had to judge whether they liked or disliked the galenic parameters. With an overall evaluation of 2.16 the test substance containing 2\% HDBM showed a good level of acceptance. First the galenic parameters such as color, scent, consistency, and spreadability were given values between 1.55 and 2.45. Further parameters such as penetration properties, and non-greasy and non-sticky characteristics of the emulsion were given values between 1.55 and 1.95. The consumption was praised as economical. The overall skin feeling was described as pleasant. In addition, caring and gentle effects were noticed. Packaging, dosage, and handling were given values between 1.59 and 2.0. All parameters except for moisturizing effect were rated lower by the placebo group (Figure 8).

\section{Discussion}

At least once in their life many people have experienced oily skin, especially as teenagers. Oily skin often has enlarged 


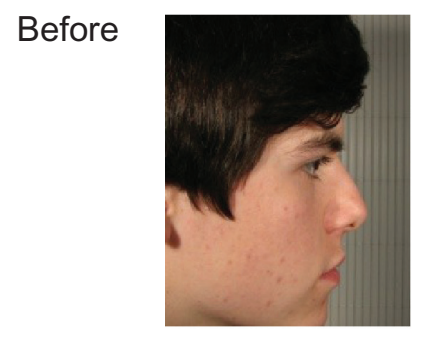

4 weeks

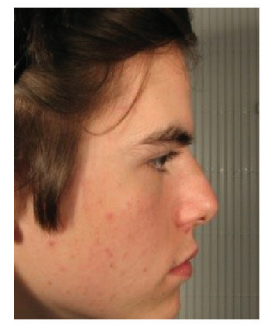

8 weeks
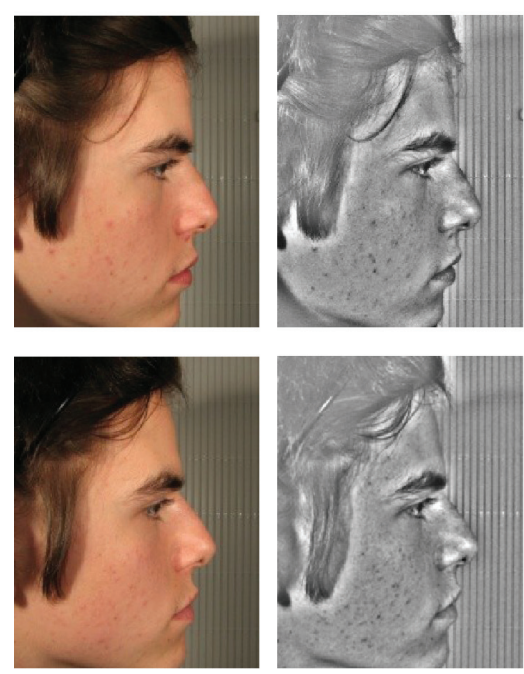
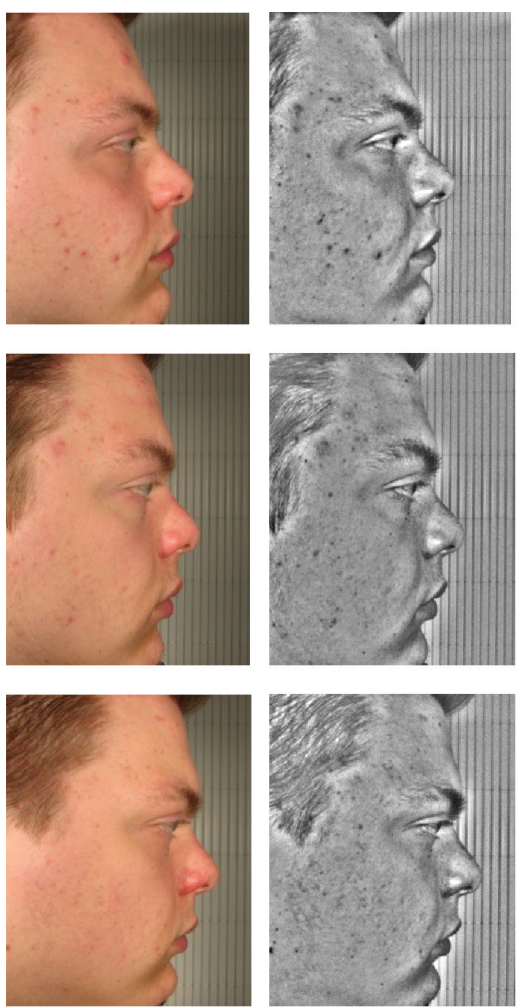

Figure 6 Representative photographs of skin improvement of two test subjects taken before and after 4 weeks and 8 weeks of treatment with $2 \%$ HDBM are shown. Notes: DatInf ${ }^{\circledR}$ UVScan/UV-Scan software was used for the visualization of skin blemishes and skin irritations (grey pictures).

pores $^{15}$ and blemishes. In addition, a comparison of data from various acne treatment studies suggested a significant correlation of sebum and acne. ${ }^{16}$

The active substance HDBM showed a very good antiseborrheic efficacy. A reduction of almost $20 \%$ in sebum excretion was achieved after 8 weeks of treatment. However, the mechanisms behind this result are not yet understood and require further investigation.
In addition, HDBM is a strong antioxidant substance, which is capable of scavenging free radicals. Almost half a century ago Lorincz postulated the lipid peroxidation theory, conceding that squalene and other skin lipid oxidation products play a central role in the induction of acne. ${ }^{17}$ Over the years more and more evidence was found which supported this hypothesis and linked free radicals and skin inflammation. It was shown in animal experiments and in

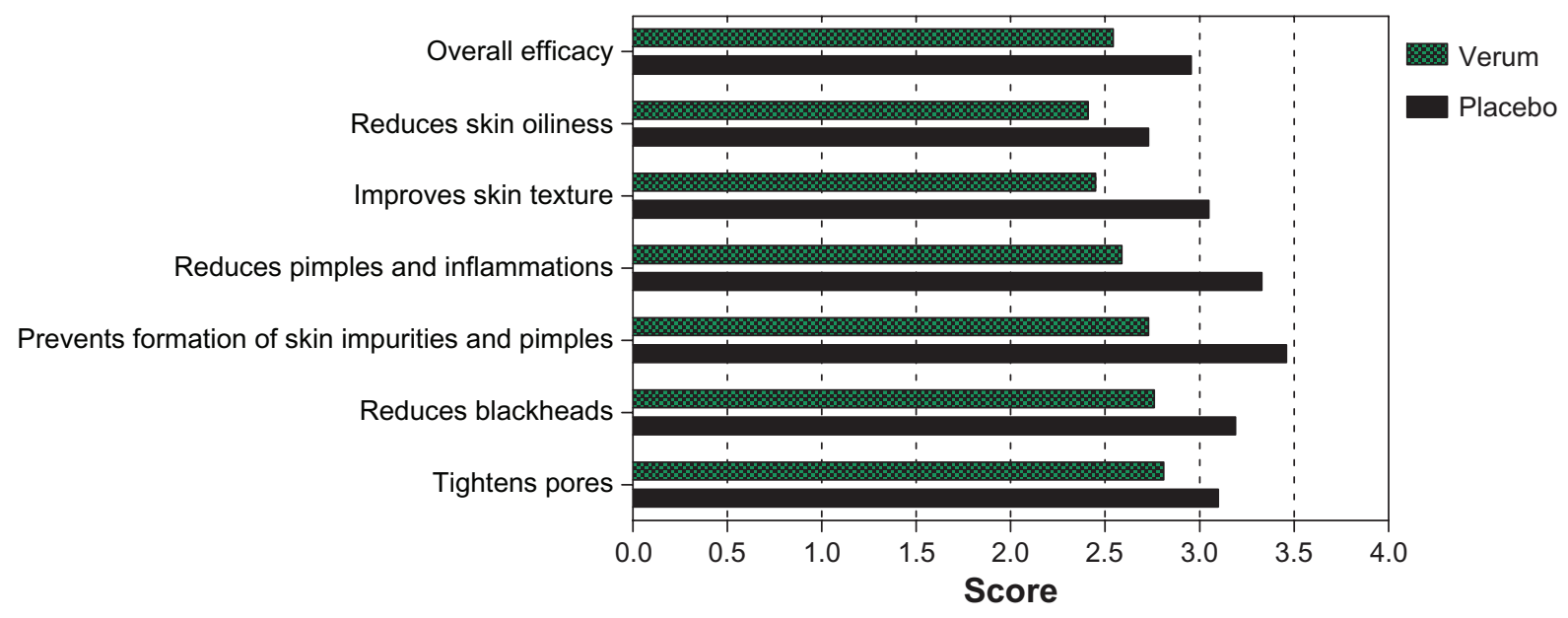

Figure 7 Subjects' self-assessment of treatment efficacy after 8 weeks with 2\% HDBM emulsion versus placebo.

Notes: Subjects rated each parameter on a 6 -point scale: $1=$ very good; 2 = good; 3 = fair; 4 = satisfactory; $5=$ unsatisfactory; $6=$ insufficient. 
humans that sebum, particularly squalene, had enhanced comedogenicity when oxidized. ${ }^{18,19}$ Thereby, the quantities of lipid peroxide in the content of comedones play an important role in the progression of comedogenesis in patients with acne vulgaris. ${ }^{20}$ Furthermore, it has been shown that one of the major antioxidative components gluthathione (GSH) was significantly lower in the stratum corneum of acne patient $\mathrm{s}^{21}$ and that the activity of both blood antioxidative enzymes superoxide dismutase and GSH-Px were also significantly lower in patients with acne. ${ }^{22}$ Only a few studies have provided results on topical application of antioxidants in the treatment of acne vulgaris. For instance, Sodium L-ascorbyl-2-phosphate, a stable vitamin $\mathrm{C}$ derivate, has been shown to suppress sebum oxidation by up to $40 \%$ and at the same time has demonstrated efficacy in the treatment of acne vulgaris. ${ }^{23,24}$ Topical $2 \%$ green tea lotions and fullerene, both agents with antioxidative activity, have shown an improvement of acne vulgaris. ${ }^{25-27}$ This research fits very well with our data, showing that HDBM, as a highly effective antioxidant significantly reduced redness and inflammation by $25 \%$. These results strongly suggest that HDBM may have an effect on the suppression of the sebum oxidation. Thus, the efficacy of HDBM is based on two pillars: sebum reduction and skin inflammation/redness reduction probably by an antioxidative mechanism.

In both groups treatment with the emulsion (verum or placebo) was well tolerated with mean scores for total intolerance reported as mild in most cases. The most common adverse event experienced by the test subjects was burning skin in the verum group (22.7\%) as well as in the placebo group $(27.8 \%)$. Blemished skin is also prone to irritation. In order to prevent a worsening of the skin condition, the problem skin needs special care. Therefore, skin care products have to be mild with excellent skin compatibility, preventing skin irritation and dryness. In this study a model formulation containing the active substance $2 \%$ HDBM was used to assess the efficacy. AEs reported in both groups suggest that the AEs are not due to the active substance but rather allow the conclusion that the formulation does not work optimally for sensitive skin. The focus of the present investigation

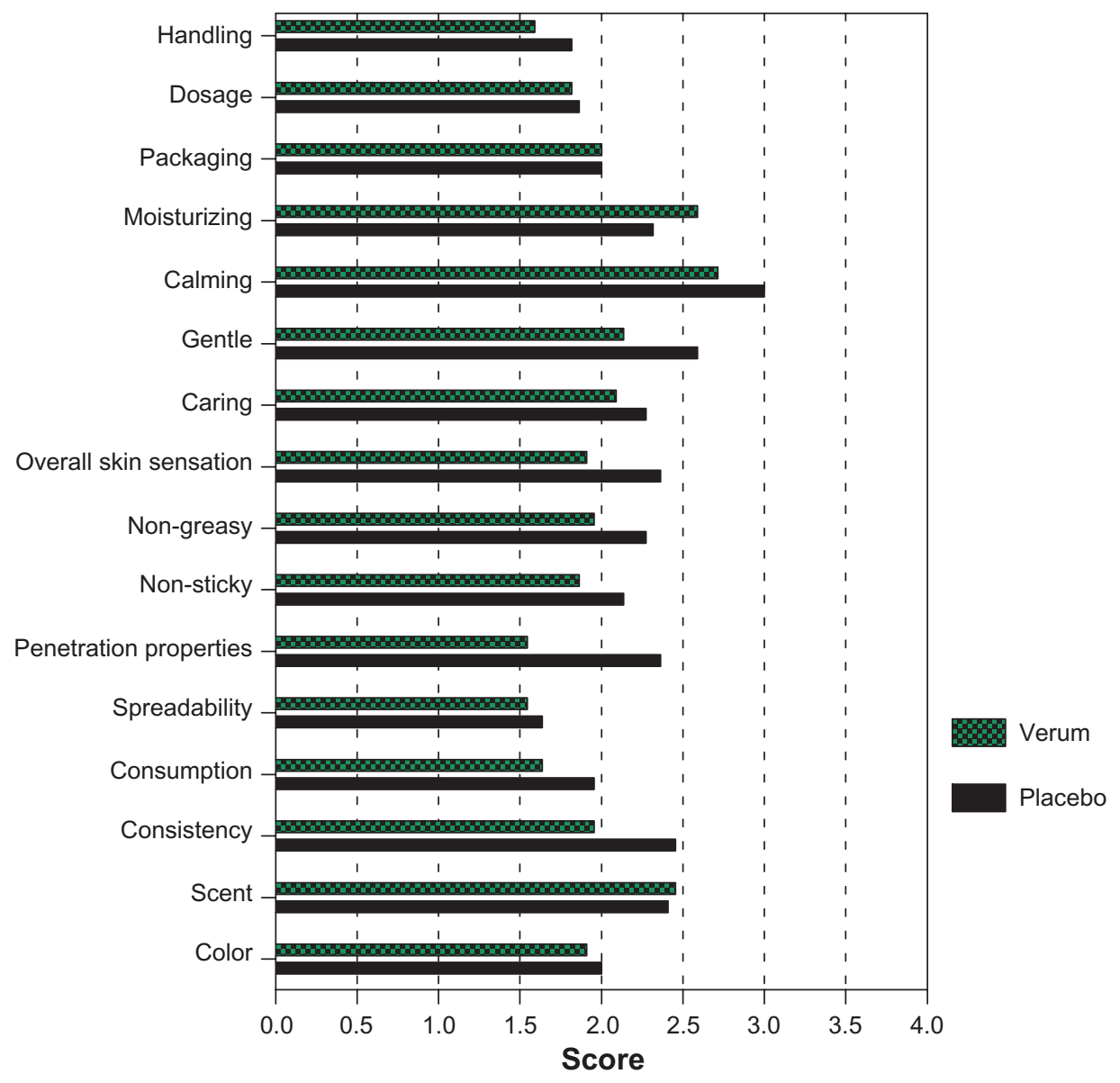

Figure 8 Subjects' self-assessment of cosmetic acceptance after 8 weeks of treatment with $2 \%$ HDBM emulsion or placebo. Notes: Subjects rated each parameter on a 6-point scale: I = very good; 2 = good; 3 = fair; 4 = satisfactory; $5=$ unsatisfactory; $6=$ insufficient. 
was not primarily on the development of an emulsion which has been optimized for sensitive skin. The emulsion matrix served more as a vehicle for the topical application of the active ingredient HDBM and to measure its effectiveness in vivo. However, the skin compatibility was assessed as far better by the verum group compared to the placebo group: a possible explanation for this could be the positive influence of the efficacy by the product. Approximately two thirds of the test subjects stated an improved skin texture and reduced skin oiliness and half of the test subjects reported a reduction of pimples and inflammation. The other self-assessed efficacy parameters did not score particularly well but still better than in the placebo group.

In conclusion, this study demonstrated that the active substance $2 \%$ HDBM has antiseborrheic and anti-inflammatory activities with minimal side effects and hence is helpful in stabilizing oily and blemished skin.

\section{Acknowledgments}

We thank Mrs M Herling for assistance and Mrs Dipl. Stat. A Grieger for the statistical evaluation of the data, and all the volunteers who made this investigation possible. The research (DT-No 136/10/10 and 036/03/11) was sponsored by Merck KGaA, Darmstadt, Germany. The sponsor participated in the discussion of the study design and provided the study emulsion.

\section{Disclosure}

The authors report no conflicts of interest in this work.

\section{References}

1. Ghodsi SZ, Orawa H, Zouboulis CC. Prevalence, severity, and severity risk factors of acne in high school pupils: a community-based study. J Invest Dermatol. 2009;129(9):2136-2141.

2. Rivera AE. Acne scarring: a review and current treatment modalities. J Am Acad Dermatol. 2008;59(4):659-676.

3. Harris HH, Downing DT, Stewart ME, Strauss JS. Sustainable rates of sebum secretion in acne patients and matched normal control subjects. J Am Acad Dermatol. 1983;8(2):200-203.

4. Powell EW, Beveridge GW. Sebum excretion and sebum composition in adolescent men with and without acne vulgaris. $\mathrm{Br} J$ Dermatol. 1970;82(3):243-249.

5. Gollnick HP, Zouboulis CC, Akamatsu H, Kurokawa I, Schulte A. Pathogenesis and pathogenesis related treatment of acne. J Dermatol. 1991;18(9):489-499.

6. Piérard-Franchimont C, Piérard GE, Saint-Léger D, Lévêque JL, Kligman AM. Comparison of the kinetics of sebum secretion in young women with and without acne. Dermatologica. 1991;183(2):120-122.

7. Bataille V, Snieder H, MacGregor AJ, Sasieni P, Spector TD. The influence of genetics and environmental factors in the pathogenesis of acne: a twin study of acne in women. J Invest Dermatol. 2002;119(6):1317-1322.
8. Chiu A, Chon SY, Kimball AB. The response of skin disease to stress: changes in the severity of acne vulgaris as affected by examination stress. Arch Dermatol. 2003;139(7):897-900.

9. Fulton JE Jr, Pay SR, Fulton JE 3rd. Comedogenicity of current therapeutic products, cosmetics, and ingredients in the rabbit ear. JAm Acad Dermatol. 1984;10(1):96-105.

10. Ottaviani M, Alestas T, Flori E, Mastrofrancesco A, Zouboulis CC, Picardo M. Peroxidated squalene induces the production of inflammatory mediators in $\mathrm{HaCaT}$ keratinocytes: a possible role in acne vulgaris. J Invest Dermatol. 2006;126(11):2430-2437.

11. Auffray B. Protection against singlet oxygen, the main actor of sebum squalene peroxidation during sun exposure, using Commiphora myrrha essential oil. Int J Cosmet Sci. 2007;29(1):23-29.

12. Rudolph T, Bühle P, Beck J, Pflücker F, Reiffen KA, Buchholz H. Hydroxy dimethoxy benzylmalonate: a novel anti-(photo)aging concept. IFSCC Magazine. 2006;9:211-216.

13. Graf R, Beck J, Rudolph T, Jung K, Herrling T, Pflücker F. Antioxidative power of formulations over life time: unique active superior than vitamins. SOFW Journal. 2008;134:52-60.

14. Chaudhuri RK, Lascu Z, Puccetti G, Deshpande AA, Paknikar SK. Design of a photostabilizer having built-in antioxidant functionality and its utility in obtaining broad-spectrum sunscreen formulations. Photochem Photobiol. 2006;82(3):823-828.

15. Kim B, Choi J, Park K, Youn S. Sebum, acne, skin elasticity, and gender difference - which is the major influencing factor for facial pores? Skin Res Technol. December 28, 2011. [Epub ahead of print.]

16. Janiczek-Dolphin N, Cook J, Thiboutot D, Harness J, Clucas A. Can sebum reduction predict acne outcome? Br J Dermatol. 2010;163(4): 683-688.

17. Lorincz AL. Human skin lipids and their relation to skin diseases. Armed Services Technical Information Report. 1965;AD647008:1-12.

18. Mills OH, Porte M, Kligman AM. Enhancement of comedogenic substances by ultraviolet radiation. Br J Dermatol. 1978;98(2):145-150.

19. Chiba K, Yoshizawa K, Makino I, Kawakami K, Onoue M. Comedogenicity of squalene monohydroperoxide in the skin after topical application. J Toxicol Sci. 2000;25(2):77-83.

20. Tochio T, Tanaka H, Nakata S, Ikeno H. Accumulation of lipid peroxide in the content of comedones may be involved in the progression of comedogenesis and inflammatory changes in comedones. $J$ Cosmet Dermatol. 2009;8(2):152-158.

21. Ikeno H, Tochio T, Tanaka H, Nakata S. Decrease in glutathione may be involved in pathogenesis of acne vulgaris. $J$ Cosmet Dermatol. 2011;10(3):240-244.

22. Basak PY, Gultekin F, Kilinc I. The role of the antioxidative defense system in papulopustular acne. J Dermatol. 2001;28(3):123-127.

23. Klock J, Ikeno H, Ohmori K, Nishikawa T, Vollhardt J, Schehlmann V. Sodium ascorbyl phosphate shows in vitro and in vivo efficacy in the prevention and treatment of acne vulgaris. Int J Cosmet Sci. 2005;27(3):171-176.

24. Woolery-Lloyd H, Baumann L, Ikeno H. Sodium L-ascorbyl2-phosphate $5 \%$ lotion for the treatment of acne vulgaris: a randomized, double-blind, controlled trial. J Cosmet Dermatol. 2010;9(1):22-27.

25. Elsaie ML, Abdelhamid MF, Elsaaiee LT, Emam HM. The efficacy of topical $2 \%$ green tea lotion in mild-to-moderate acne vulgaris. J Drugs Dermatol. 2009;8(4):358-364.

26. Xu Y, Zhang JJ, Xiong L, Zhang L, Sun D, Liu H. Green tea polyphenols inhibit cognitive impairment induced by chronic cerebral hypoperfusion via modulating oxidative stress. $J$ Nutr Biochem. 2010;21(8):741-748.

27. Inui S, Aoshima H, Nishiyama A, Itami S. Improvement of acne vulgaris by topical fullerene application: unique impact on skin care. Nanomedicine. 2011;7(2):238-241. 


\section{Publish your work in this journal}

Clinical, Cosmetic and Investigational Dermatology is an international, peer-reviewed, open access, online journal that focuses on the latest clinical and experimental research in all aspects of skin disease and cosmetic interventions. All areas of dermatology will be covered; contributions will be welcomed from all clinicians and basic science researchers globally. This journal is indexed on CAS. The manuscript management system is completely online and includes a very quick and fair peer-review system, which is all easy to use. Visit http://www.dovepress.com/testimonials.php to read real quotes from published authors.

Submit your manuscript here: http://www.dovepress.com/clinical-cosmetic-and-investigational-dermatology-journal 